\title{
OPTIMAL CHANNEL SWITCHING FOR AVERAGE CAPACITY MAXIMIZATION
}

\author{
Ahmet Dundar Sezer*, Sinan Gezici*, and Hazer Inaltekin ${ }^{\sharp}$ \\ * Dept. of Electrical and Electronics Engineering, Bilkent University, 06800, Ankara, Turkey \\ $\sharp$ Dept. of Electrical and Electronics Engineering, Antalya International University, Antalya, Turkey
}

\begin{abstract}
Optimal channel switching is proposed for average capacity maximization in the presence of average and peak power constraints. A necessary and sufficient condition is derived in order to determine when the proposed optimal channel switching approach can or cannot outperform the optimal single channel approach, which performs no channel switching. Also, it is stated that the optimal channel switching solution can be realized by channel switching between at most two different channels. In addition, a low-complexity optimization problem is derived in order to obtain the optimal channel switching solution. Numerical examples are provided to exemplify the derived theoretical results.
\end{abstract}

Index Terms - Channel switching, capacity, time-sharing.

\section{INTRODUCTION}

Recently, benefits of randomization (or, time-sharing) have been studied for various detection and estimation problems in the literature [1]-[13]. For instance, in the context of noise enhanced detection and estimation, an additive "noise" component that is realized by a randomization among a certain number of signal levels can be injected into the input of a suboptimal detector or estimator for performance enhancement [1]-[5]. Also, error performance of power constrained communications systems that operate in non-Gaussian channels can be improved via stochastic signaling, which involves modeling the signal values transmitted for each information symbol as random variables $[8,9]$. It is shown that an optimal stochastic signal can be represented by a randomization of no more than three different signal values under second and fourth moment constraints [8].

Error performance of some communications systems that operate over additive time-invariant noise channels can be enhanced via detector randomization, which involves the use of multiple detectors at the receiver with certain probabilities $[3,10,14,15,16]$. In [3], an average power constrained binary communication system is studied, and randomization between two antipodal signal pairs and the corresponding MAP detectors is considered. Significant performance improvements are reported as a result of detector randomization in the presence of symmetric Gaussian mixture noise over a range of average power constraint values. In [10], the results in [3] and
[9] are extended by considering an average power constrained $M$-ary communications system that can employ both detector randomization and stochastic signaling over an additive noise channel with a known distribution. It is obtained that the joint optimization of the transmitted signals and the detectors at the receiver results in a randomization between at most two MAP detectors corresponding to two deterministic signal constellations.

In the presence of multiple channels between a transmitter and a receiver, it may be advantageous to perform channel switching; that is, to transmit over one channel for a certain fraction of time, and then switch to another channel during the next transmission period even if the channel statistics are not varying with time $[6,17,18,19]$. In [6], it is shown that the optimum performance under an average power constraint can be achieved by time sharing between no more than two channels and power levels. In addition, [19] considers the channel switching problem in the presence of stochastic signaling, and obtains the optimal strategy, which can involve either transmitting over a single channel with deterministic or stochastic signaling, or channel switching between two channels with deterministic signaling.

Although the optimal channel switching problem is studied in [6] and [19] in terms of average probability of error minimization, no studies in the literature have considered the channel switching problem for capacity maximization. In this study, we formulate the optimal channel switching problem for capacity maximization under average and peak power constraints, and derive a necessary and sufficient condition for the proposed channel switching approach to achieve a higher average capacity than the no channel switching approach. In addition, it is stated that the optimal solution to the channel switching problem results in channel switching between at most two different channels, and an approach is proposed to obtain the optimal channel switching strategy with low computational complexity. Numerical examples are presented to illustrate the theoretical results.

\section{OPTIMAL CHANNEL SWITCHING}

Consider a communications system in which a transmitter and a receiver are connected via $K$ different channels as shown in Fig. 1. The channels are modeled as additive Gaussian noise channels with possibly different bandwidths and noise lev- 


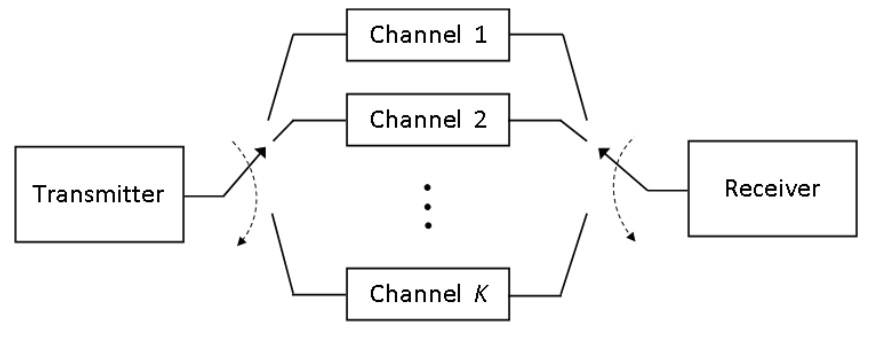

Fig. 1. Block diagram of a communication system in which transmitter and receiver can switch between $K$ channels.

els. The transmitter and the receiver can switch or time-share among these $K$ channels to improve the capacity of the communications system. A relay at the transmitter controls access to the channels in such a way that only one of the channels can be employed for symbol transmission at any given time. The transmitter and the receiver are assumed to be synchronized so that the receiver knows which channel is being utilized. In practice, this assumption can be realized by employing a communication protocol that allocates the first $N_{s, 1}$ symbols in the payload for channel 1 , the next $N_{s, 2}$ symbols in the payload for channel 2, and so on. The information on the number of symbols for different channels can be included in the header of a communications packet $[10,19]$.

A motivating example for a system as in Fig. 1 is a cognitive radio system, in which secondary users can utilize multiple available frequency bands in the spectrum [20, 21]. In such a scenario, optimal channel switching investigated in this study can be employed in order to maximize the average capacity of secondary users. The proposed system has also the potential to improve capacity in emerging open-access $K$-tier heterogeneous wireless networks $[22,23]$.

Let $B_{i}$ and $N_{i} / 2$ denote, respectively, the bandwidth and the constant power spectral density level of the additive Gaussian noise corresponding to channel $i$ for $i \in\{1, \ldots, K\}$. Then, the capacity of channel $i$ can be expressed as

$$
C_{i}(P)=B_{i} \log _{2}\left(1+\frac{P}{N_{i} B_{i}}\right) \text { bits/sec }
$$

where $P$ denotes the average transmit power [24].

In this study, the aim is to obtain the optimal channel switching strategy that maximizes the average capacity of the communication system in Fig. 1 under average and peak power constraints. In order to formulate such a problem, we first define $\lambda_{1}, \ldots, \lambda_{K}$ as the channel switching (timesharing) factors, where $\lambda_{i}$ is the fraction of time when channel $i$ is used, with $\lambda_{i} \geq 0$ for $i=1, \ldots, K$, and $\sum_{i=1}^{K} \lambda_{i}=1$. Then, we propose the following optimal channel switching problem for capacity maximization:

$$
\begin{aligned}
\max _{\left\{\lambda_{i}, P_{i}\right\}_{i=1}^{K}} & \sum_{i=1}^{K} \lambda_{i} C_{i}\left(P_{i}\right) \\
\text { subject to } & \sum_{i=1}^{K} \lambda_{i} P_{i} \leq P_{\mathrm{av}}, P_{i} \in\left[0, P_{\mathrm{pk}}\right], \forall i \in\{1, \ldots, K\} \\
& \sum_{i=1}^{K} \lambda_{i}=1, \lambda_{i} \geq 0, \forall i \in\{1, \ldots, K\}
\end{aligned}
$$

where $C_{i}\left(P_{i}\right)$ is as defined in (1) with $P_{i}$ being the average transmit power allocated to channel $i, P_{\mathrm{pk}}$ denotes the peak power constraint, and $P_{\mathrm{av}}$ is the average power constraint for the transmitter. It is assumed that $P_{\mathrm{av}}<P_{\mathrm{pk}}$.

In general, it can be challenging to obtain the optimal channel switching strategy by directly solving the optimization problem in (2). Therefore, we first try to obtain a simpler version of (2), which leads to the same optimal channel switching solution. To that aim, the following proposition presents an alternative optimization problem, the solution of which achieves the same maximum average capacity as (2). (The proofs of the propositions are not presented due to the space limitation.)

Proposition 1: The solution of the following optimization problem results in the same maximum value as the one in (2):

$$
\begin{aligned}
\max _{\left\{\nu_{i}, P_{i}\right\}_{i=1}^{K}} & \sum_{i=1}^{K} \nu_{i} C_{\max }\left(P_{i}\right) \\
\text { subject to } & \sum_{i=1}^{K} \nu_{i} P_{i} \leq P_{\mathrm{av}}, P_{i} \in\left[0, P_{\mathrm{pk}}\right], \forall i \in\{1, \ldots, K\} \\
& \sum_{i=1}^{K} \nu_{i}=1, \nu_{i} \geq 0, \forall i \in\{1, \ldots, K\}
\end{aligned}
$$

where $C_{\max }(P)$ is defined as

$$
C_{\max }(P)=\max \left\{C_{1}(P), \ldots, C_{K}(P)\right\} .
$$

The importance of Proposition 1 is related to the fact that the alternative optimization problem in (3), which achieves the same maximum average capacity as the original problem in (2), facilitates detailed theoretical investigation of the optimal channel switching strategy, as discussed in the following.

In order to investigate the improvements that can be achieved via channel switching, the case of no channel switching is considered as a reference algorithm. In the absence of channel switching, the best channel is selected and all the available transmit power is used over that channel. In that case, the achieved maximum capacity can be expressed as $C_{\max }\left(P_{\mathrm{av}}\right)$, where $C_{\max }$ is as defined in (4), and the best channel is the one with the index $\arg \max _{l \in\{1, \ldots, K\}} C_{l}\left(P_{\mathrm{av}}\right)$. (In the case of multiple best channels, any of them can be 
chosen to achieve $\left.C_{\max }\left(P_{\mathrm{av}}\right).\right)^{1}$ This approach is called the optimal single channel algorithm in the following.

In the next proposition, a necessary and sufficient condition is presented for the optimal channel switching approach to have the same performance as the optimal single channel algorithm.

Proposition 2: Assume that $C_{\max }(P)$ in (4) is first-order continuously differentiable in an interval around $P_{\mathrm{av}}$. Then, the optimal channel switching and the optimal single channel algorithms achieve the same maximum average capacity if and only if

$$
\left(x-P_{\mathrm{av}}\right) \frac{B_{i^{*}} \log _{2} e}{N_{i^{*}} B_{i^{*}}+P_{\mathrm{av}}} \geq C_{\mathrm{max}}(x)-C_{\mathrm{max}}\left(P_{\mathrm{av}}\right)
$$

for all $x \in\left[0, P_{\mathrm{pk}}\right]$, where $i^{*}=\arg \max _{i \in\{1, \ldots, K\}} C_{i}\left(P_{\mathrm{av}}\right)$.

Based on Proposition 2, it can be determined whether channel switching can improve the average capacity of the system compared to the no channel switching case. For example, if the condition in (5) is satisfied for all $x \in\left[0, P_{\mathrm{pk}}\right]$ in a given system, then it is concluded that the optimal single channel algorithm has the same performance as the optimal channel switching algorithm; that is, there is no need for channel switching. In that case, the maximum average channel capacity is obtained as $C_{\max }\left(P_{\mathrm{av}}\right)$. On the other hand, if there exist some $x \in\left[0, P_{\mathrm{pk}}\right]$ for which the condition in (5) is not satisfied, then the optimal channel switching algorithm is guaranteed to achieve a higher average capacity than $C_{\max }\left(P_{\text {av }}\right)$.

In Proposition 2, it is assumed that $C_{\max }(P)$ in (4) is firstorder continuously differentiable in an interval around $P_{\text {av }}$. If this condition is not satisfied, then it is guaranteed that performance improvements can be obtained via channel switching, as stated in the following proposition.

Proposition 3: If the first-order derivative of $C_{\max }(P)$ in (4) is discontinuous at $P=P_{\mathrm{av}}$, then the optimal channel switching algorithm outperforms the optimal single channel algorithm.

When the optimal channel switching algorithm is guaranteed to achieve a higher average capacity than the optimal single channel algorithm (which can be deduced from Proposition 2 or Proposition 3), the optimization problem in (2) or (3) needs to be solved in order to calculate the maximum average capacity of the system, which involves a search over a $2 K$ dimensional space. However, the following proposition states that the optimal solution can be obtained by switching between no more than two different channels, and the resulting optimal strategy can be found via a search over a twodimensional space.

Proposition 4: The optimal solution of (2) results in channel switching between at most two channels, and the maximum average capacity achieved is calculated as $\lambda^{*} C_{\max }\left(P_{1}^{*}\right)+\left(1-\lambda^{*}\right) C_{\max }\left(P_{2}^{*}\right)$, where $P_{1}^{*}$ and $P_{2}^{*}$ are the

\footnotetext{
${ }^{1}$ From (1) and (4), it can be shown that $C_{\max }(P)$ is a monotone increasing and continuous function of $P$. Hence, when a single channel is used (i.e. no channel switching), it is optimal to utilize all the available power, $P_{\mathrm{av}}$.
}

solutions of the following problem:

$$
\max _{\substack{P_{1} \in\left(P_{\mathrm{av}}, P_{\mathrm{pk}}\right] \\ P_{2} \in\left[0, P_{\mathrm{av}}\right]}} \frac{P_{\mathrm{av}}-P_{2}}{P_{1}-P_{2}} C_{\max }\left(P_{1}\right)+\frac{P_{1}-P_{\mathrm{av}}}{P_{1}-P_{2}} C_{\max }\left(P_{2}\right)
$$

and $\lambda^{*}$ is given by $\lambda^{*}=\left(P_{\mathrm{av}}-P_{2}^{*}\right) /\left(P_{1}^{*}-P_{2}^{*}\right)$.

Once $\lambda^{*}, P_{1}^{*}$, and $P_{2}^{*}$ are obtained as in Proposition 4 , the optimal channel switching strategy can be specified as follows: Switch between channel $i$ and channel $j$ with channel switching (time-sharing) factors of $\lambda^{*}$ and $1-\lambda^{*}$, respectively, where

$$
\begin{aligned}
& i=\underset{l \in\{1, \ldots, K\}}{\arg \max } C_{l}\left(P_{1}^{*}\right) \\
& j=\underset{l \in\{1, \ldots, K\}}{\arg \max } C_{l}\left(P_{2}^{*}\right) .
\end{aligned}
$$

Overall, the solution of the proposed optimal channel switching problem can be obtained as follows: First, $C_{\max }(P)$ in (4) is calculated for the given system parameters. If the first-order derivative of $C_{\max }(P)$ is continuous at $P_{\text {av }}$ and the condition in Proposition 2 is satisfied, then there is no need for channel switching (i.e., the single channel approach is optimal). Otherwise, the optimal solution involves time-sharing between two channels, which can be obtained as described in the previous paragraph and Proposition 4. When the single channel approach is optimal, the optimal solution of (2) can be expressed as $\lambda_{i^{*}}=1, P_{i^{*}}=P_{\mathrm{av}}$, and $\lambda_{j}=P_{j}=0$ for all $j \in\{1, \ldots, K\} \backslash\left\{i^{*}\right\}$, where $i^{*}=\arg \max _{i \in\{1, \ldots, K\}} C_{i}\left(P_{\mathrm{av}}\right)$. In that case, the maximum average capacity becomes $C_{\max }\left(P_{\mathrm{av}}\right)$.

It should be noted that the computational complexity of the optimization problem in (6) depends on the number of channels, $K$, only through $C_{\max }$ in (4), and the dimension of the search space is always two irrespective of the number of channels. Therefore, Proposition 4 can provide a significant simplification of the original formulation in (2), which requires a search over a $2 K$ dimensional space.

\section{NUMERICAL RESULTS}

In this section, numerical examples are presented in order to investigate the proposed optimal channel switching approach and to compare it against the optimal single channel approach. Consider a scenario with $K=3$ channels with the following bandwidths and noise levels (see (1)): $B_{1}=1 \mathrm{MHz}$, $B_{2}=5 \mathrm{MHz}, B_{3}=10 \mathrm{MHz}, N_{1}=10^{-12} \mathrm{~W} / \mathrm{Hz}, N_{2}=$ $10^{-11} \mathrm{~W} / \mathrm{Hz}$, and $N_{3}=10^{-11} \mathrm{~W} / \mathrm{Hz}$. Assume that the peak power constraint in (2) is set to $P_{\mathrm{pk}}=0.1 \mathrm{~mW}$. In Fig 2, the capacity of each channel is plotted as a function of power based on the capacity expression in (1).

In Fig. 3, the performance of the proposed optimal channel switching algorithm is compared against that of the optimal single channel algorithm. As discussed in the previous section, the optimal single channel algorithm achieves a capacity of $C_{\max }\left(P_{\mathrm{av}}\right)$, which is $C_{\max }\left(P_{\mathrm{av}}\right)=$ 


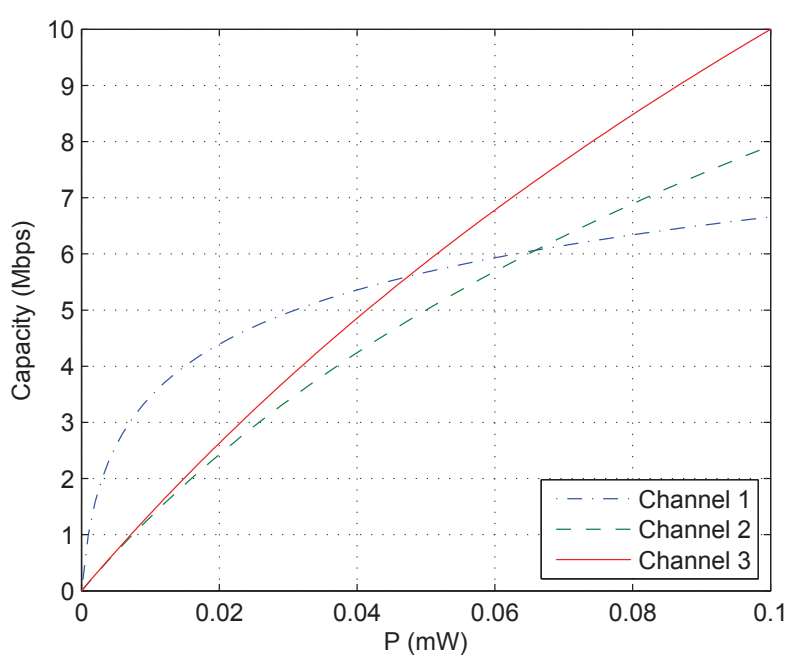

Fig. 2. Capacity of each channel versus power.

$\max \left\{C_{1}\left(P_{\mathrm{av}}\right), C_{2}\left(P_{\mathrm{av}}\right), C_{3}\left(P_{\mathrm{av}}\right)\right\}$ in the considered scenario. It is noted from Fig 2 and Fig. 3 that $C_{\max }\left(P_{\mathrm{av}}\right)=$ $C_{1}\left(P_{\mathrm{av}}\right)$ for $P_{\mathrm{av}} \in(0,0.048) \mathrm{mW}$ and $C_{\mathrm{max}}\left(P_{\mathrm{av}}\right)=C_{3}\left(P_{\mathrm{av}}\right)$ for $P_{\mathrm{av}} \in[0.048,0.1] \mathrm{mW}$; that is, channel 1 is the best channel up to $P_{\text {av }}=0.048 \mathrm{~mW}$, and channel 3 is the best after that power level. From Fig. 3, it is also observed that the proposed optimal channel switching algorithm outperforms the optimal single channel algorithm for $P_{\mathrm{av}} \in[0.02,0.1] \mathrm{mW}$, and the two algorithms have the same performance for $P_{\mathrm{av}}<0.02 \mathrm{~mW}$. These regions can also be obtained by checking the necessary and sufficient condition in Proposition 2 (see (5)), which is satisfied for all $x \in[0,0.1] \mathrm{mW}$ for $P_{\text {av }}<0.02 \mathrm{~mW}$, and is not satisfied for some $x \in[0,0.1] \mathrm{mW}$ for $P_{\mathrm{av}} \in[0.02,0.1] \mathrm{mW}$. $^{2}$ Also, in accordance with Proposition 3 , it is observed that the optimal channel switching algorithm outperforms the optimal single channel algorithm at $P_{\text {av }}=0.048 \mathrm{~mW}$, which corresponds to a discontinuity point for the first-order derivative of $C_{\max }(P)$.

In order to provide a detailed investigation of the optimal channel switching strategy, Table 1 presents the optimal channel switching solutions for various values of $P_{\mathrm{av}}$. As in (6)-(8), the optimal solution is represented by parameters $\lambda^{*}$, $P_{1}^{*}, P_{2}^{*}, i$, and $j$, meaning that channel $i$ is used with channel switching factor $\lambda^{*}$ and power $P_{1}^{*}$ and channel $j$ is used with channel switching factor $1-\lambda^{*}$ and power $P_{2}^{*}$. From the table, it is observed that the optimal solution reduces to the optimal single channel solution for $P_{\mathrm{av}}=0.01 \mathrm{~mW}$ (in which case channel 1 is used all the time), and it involves switching ("randomization") between channel 1 and channel 3 for larger values of $P_{\mathrm{av}}$. This observation is also consistent with Fig. 3, which illustrates improvement via channel switching for $P_{\mathrm{av}}>0.02 \mathrm{~mW}$.

Based on this numerical example, an intuitive explanation can be provided about the benefits of channel switching and why the optimal channel switching strategy involves

\footnotetext{
${ }^{2}$ The details of the calculations are not shown due to the space limitation.
}

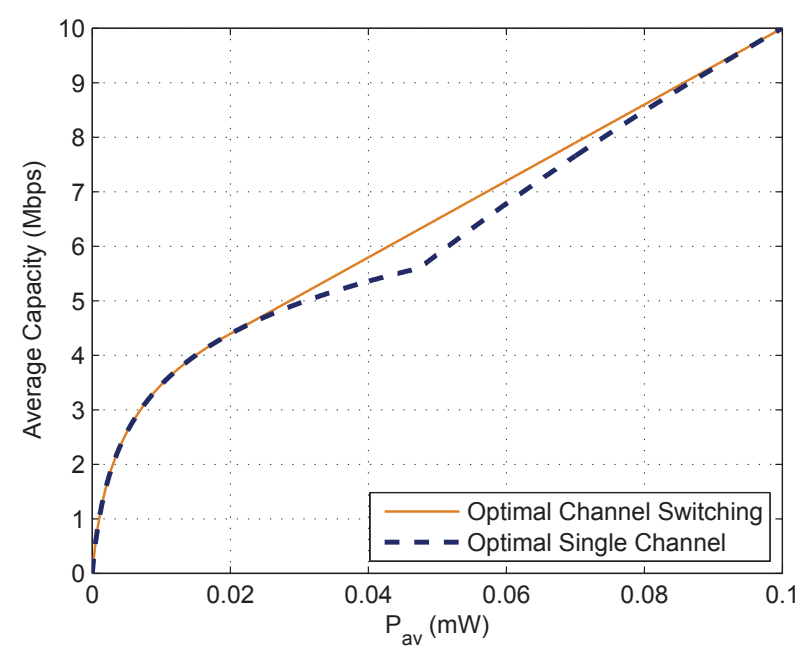

Fig. 3. Average capacity versus average power limit for the optimal channel switching and the optimal single channel approaches.

\begin{tabular}{|c||c|c|c||c|c|c|}
\hline$P_{\mathrm{av}}(\mathrm{mW})$ & $\lambda^{*}$ & $P_{1}^{*}$ & $i$ & $\left(1-\lambda^{*}\right)$ & $P_{2}^{*}$ & $j$ \\
\hline 0.01 & 1 & 0.01 & 1 & - & - & - \\
0.03 & 0.871 & 0.02 & 1 & 0.129 & 0.1 & 3 \\
0.05 & 0.622 & 0.02 & 1 & 0.378 & 0.1 & 3 \\
0.07 & 0.373 & 0.02 & 1 & 0.627 & 0.1 & 3 \\
0.09 & 0.124 & 0.02 & 1 & 0.876 & 0.1 & 3 \\
\hline
\end{tabular}

Table 1. Optimal channel switching strategy, which employs channel $i$ for $100 \lambda^{*}$ percent of time with power $P_{1}^{*}$, and channel $j$ for $100\left(1-\lambda^{*}\right)$ percent of time with power $P_{2}^{*}$.

switching between no more than two channels. In the absence of channel switching, the optimal capacity is given by $C_{\max }\left(P_{\mathrm{av}}\right)$, whereas via channel switching, the upper boundary of the convex hull of $C_{\max }\left(P_{\mathrm{av}}\right)$ can also be achieved (see Fig. 3). Since the upper boundary of the convex hull is always formed by a convex combination of two different points, no more than two different channels are needed to achieve the optimal capacity.

\section{CONCLUDING REMARKS}

In this study, optimal channel switching has been proposed for average capacity maximization in the presence of average and peak power constraints. A necessary and sufficient condition has been derived for specifying whether the proposed optimal channel switching approach can or cannot outperform the optimal single channel approach. In addition, the optimal channel switching solution has been shown to be realized by channel switching between at most two different channels, and a low-complexity optimization problem has been obtained to calculate the optimal channel switching solution. Numerical examples have been presented and intuitive explanations about the benefits of channel switching have been provided. 


\section{REFERENCES}

[1] H. Chen, P. K. Varshney, S. M. Kay, and J. H. Michels, "Theory of the stochastic resonance effect in signal detection: Part I-Fixed detectors," IEEE Trans. Sig. Processing, vol. 55, no. 7, pp. 3172-3184, July 2007.

[2] H. Chen, P. K. Varshney, and J. H. Michels, "Noise enhanced parameter estimation," IEEE Trans. Sig. Processing, vol. 56, no. 10, pp. 5074-5081, Oct. 2008.

[3] A. Patel and B. Kosko, "Optimal noise benefits in Neyman-Pearson and inequality-constrained signal detection," IEEE Trans. Sig. Processing, vol. 57, no. 5, pp. 1655-1669, May 2009.

[4] S. Bayram and S. Gezici, "Noise-enhanced $M$-ary hypothesis-testing in the minimax framework," in Proc. International Conference on Signal Processing and Commun. Systems, Omaha, Nebraska, Sep. 2009, pp. 31-36.

[5] S. Bayram, S. Gezici, and H. V. Poor, "Noise enhanced hypothesis-testing in the restricted Bayesian framework," IEEE Trans. Sig. Processing, vol. 58, no. 8, pp. 3972-3989, Aug. 2010.

[6] M. Azizoglu, "Convexity properties in binary detection problems," IEEE Trans. Inform. Theory, vol. 42, no. 4, pp. 1316-1321, July 1996.

[7] S. Loyka, V. Kostina, and F. Gagnon, "Error rates of the maximum-likelihood detector for arbitrary constellations: Convex/concave behavior and applications," IEEE Trans. Inform. Theory, vol. 56, no. 4, pp. 19481960, April 2010.

[8] C. Goken, S. Gezici, and O. Arikan, "Optimal stochastic signaling for power-constrained binary communications systems," IEEE Trans. Wireless Commun., vol. 9, no. 12, pp. 3650-3661, Dec. 2010.

[9] C. Goken, S. Gezici, and O. Arikan, "Optimal signaling and detector design for power-constrained binary communications systems over non-Gaussian channels," IEEE Commun. Letters, vol. 14, no. 2, pp. 100-102, Feb. 2010.

[10] B. Dulek and S. Gezici, "Detector randomization and stochastic signaling for minimum probability of error receivers," IEEE Trans. Commun., vol. 60, no. 4, pp. 923-928, Apr. 2012.

[11] B. Dulek, S. Gezici, and O. Arikan, "Convexity properties of detection probability under additive Gaussian noise: Optimal signaling and jamming strategies," IEEE Trans. Signal Process., vol. 61, no. 13, pp. 3303-3310, July 2013.
[12] M. E. Tutay, S. Gezici, and O. Arikan, "Optimal detector randomization for multiuser communications systems," accepted to IEEE Trans. Commun., 2013.

[13] B. Dulek, N. D. Vanli, S. Gezici, and P. K. Varshney, "Optimum power randomization for the minimization of outage probability," IEEE Trans. Wirel. Commun., vol. 12, no. 9, pp. 4627-4637, Sep. 2013.

[14] H. Chen, P. K. Varshney, S. M. Kay, and J. H. Michels, "Theory of the stochastic resonance effect in signal detection: Part II-Variable detectors," IEEE Trans. Sig. Processing, vol. 56, no. 10, pp. 5031-5041, Oct. 2007.

[15] B. Dulek and S. Gezici, "Optimal stochastic signal design and detector randomization in the Neyman-Pearson framework," in 37th IEEE Int. Conf. Acous., Speech, Signal Process. (ICASSP), Kyoto, Japan, Mar. 25-30 2012, pp. 3025-3028.

[16] E. L. Lehmann, Testing Statistical Hypotheses, Chapman \& Hall, New York, 2 edition, 1986.

[17] Y. Ma and C. C. Chai, "Unified error probability analysis for generalized selection combining in Nakagami fading channels," IEEE J. Select. Areas Commun., vol. 18, no. 11, pp. 2198-2210, Nov. 2000.

[18] J. A. Ritcey and M. Azizoglu, "Performance analysis of generalized selection combining with switching constraints," IEEE Commun. Letters, vol. 4, no. 5, pp. 152154, May 2000.

[19] B. Dulek, P. K. Varshney, M. E. Tutay, and S. Gezici, "Optimal channel switching in the presence of stochastic signaling," in Proc. IEEE International Symposium on Information Theory Proceedings (ISIT), July 2013, pp. 2189-2193.

[20] J. Mitola and G. Q. Maguire, “Cognitive radio: Making software radios more personal," IEEE Personal Commun. Mag., vol. 6, no. 4, pp. 13-18, Aug. 1999.

[21] S. Gezici, H. Celebi, H. V. Poor, and H. Arslan, "Fundamental limits on time delay estimation in dispersed spectrum cognitive radio systems," IEEE Trans. Wirel. Commun., vol. 8, no. 1, pp. 78-83, Jan. 2009.

[22] J. G. Andrews, H. Claussen, M. Dohler, S. Rangan, and M. C. Reed, "Femtocells: Past, present, and future," IEEE J. Sel. Areas Commun., vol. 30, no. 3, pp. 497508, Apr. 2012.

[23] A. Ghosh, et.al., "Heterogeneous cellular networks: From theory to practice," IEEE Trans. Commun. Mag., vol. 50, no. 6, pp. 54-64, June 2012.

[24] Thomas M. Cover and Joy A. Thomas, Elements of Information Theory, Wiley-Interscience, 1991. 